Artigo / Article

\title{
Síndrome mielodisplásica secundária à quimio ou radioterapia - SMD relacionada a tratamento
}

\author{
Myelodysplastic syndrome secondary to chemo - and radiotherapy MDS related to the treatment
}

Ronald F. Pinheiro ${ }^{1}$

Maria de Lourdes L. F. Chauffaille ${ }^{2}$

\begin{abstract}
O efeito leucemogênico dos agentes quimioterápicos após o tratamento para neoplasias é bem conhecido. Síndrome mielodisplásica secundária a quimio ou radioterapia, também denominada relacionada à terapia (SMD-t), geralmente ocorre quatro a sete anos após a exposição inicial ao agente quimio ou radioterápico, acomete habitualmente pacientes jovens, apresenta alta incidência de transformação para leucemia mielóide aguda (LMA), está associada a citopenias severas, displasia das três linhagens, celularidade medular reduzida e fibrose, e anormalidades citogenéticas em até $80 \%$ dos casos. As anormalidades mais freqüentes envolvem os cromossomos 5 e 7. No tocante à quimioterapia, os agentes alquilantes são as drogas mais comumente associadas com SMD-t. Quimioterápicos em altas doses usados como parte de regimes de condicionamento para transplantes de medula óssea e radioterapia, além dos esquemas COPP/ABV e BEACOPP, estão associados com SMD-t. Recentemente, drogas como azatioprina, cladribina e rituximab também foram relacionadas à SMD-t. Devido ao aumento da sobrevida de pacientes acometidos por neoplasias malignas, a SMD-t surge como efeito mutagênico desses tratamentos e confere prognóstico desfavorável. Rev. bras. hematol. hemoter. 2006;28(3):201-203.
\end{abstract}

Palavras-chave: Síndrome mielodisplásica secundária; SMD relacionada a quimioterapia; SMD-t.

\section{Introdução}

Síndrome mielodisplásica secundária à quimioterapia (SMD-t) é uma doença clonal com curso clínico mais agressivo que a SMD primária, com incidência variável a depender da população estudada, esquema quimioterápico utilizado e tempo de seguimento dos pacientes.

A incidência de SMD-t vem aumentando nos últimos anos devido aos esquemas quimioterápicos mais intensivos (com objetivos curativos), assim como aos regimes de condicionamento relacionados aos transplantes de medula óssea. De uma maneira geral, as drogas associadas ao desenvolvimento de SMD-t são os agentes alquilantes, além da radioterapia.
Tem sido observado risco aumentado de SMD-t em pacientes seguidos por longa data com doença de Hodgkin, linfoma não-Hodgkin, mieloma múltiplo e neoplasias gastrintestinais, especialmente aqueles que receberam tratamento com metil-CCNU. O risco para pacientes com doença de Hodgkin é estimado em $4 \%$ a $6 \%$ e para pacientes tratados de mieloma múltiplo de até $17 \%$. O número de casos relacionados ao tratamento de neoplasias de mama, ovário, testículo e pequenas células de pulmão é considerado relativamente baixo com o uso de quimioterápicos; porém, quando há a associação de radioterapia local, a incidência aumenta.

A SMD-t geralmente se desenvolve quatro a sete anos após a exposição a quimio ou radioterápicos e corresponde a cerca de $10 \%$ do total de casos de SMD. Na disciplina de

${ }^{1}$ Aluno de Pós-graduação, Unifesp/EPM.

${ }^{2}$ Profa. adjunta. Livre-docente, assessora médica do Fleury - Centro de Medicina Diagnóstica.

Disciplina de Hematologia e Hemoterapia, Unifesp/EPM.

Correspondência: Maria de Lourdes L. F. Chauffaille

Rua Botucatu $740,3^{\circ}$ andar

04023-900 - São Paulo, SP

Tel.: 55-11-5576-4240 - E-mail: chauffaill@hemato.epm.br 
Hematologia e Hemoterapia da Unifesp-EPM, as SMD-t perfazem 5\% do total de casos de SMD, e o uso de agentes alquilantes é responsável por metade desses casos.

A título de exemplo, dois casos são aqui descritos:

Caso 1: Paciente de sexo feminino, 54 anos, teve diagnóstico de neoplasia de laringe e recebeu tratamento à base de alquilantes e radioterapia local no ano de 1999. Em 2003, apresentou leucopenia seguida de pancitopenia. No mielograma (MO) foi observada displasia severa nas três linhagens, na biópsia de medula óssea (BMO) havia agrupamentos de células imaturas (ALIPS) e no cariótipo foram detectadas: a deleção do cromossomo 5, monossomia do cromossomo 7 , trissomia do cromossomo 19 e a presença de duplo-minutos (pequenos fragmentos cromossômicos decorrentes de amplificação gênica), portanto considerado como cariótipo complexo. Foi feito o diagnóstico de SMD-t e estabalecido o prognóstico como desfavorável.

Caso 2: Paciente de sexo feminino, 40 anos, com antecedente de neoplasia de mama tratada há dois anos com inibidor de topoisomerase e radioterapia local, apresentava trombocitopenia $\left(80.000 / \mathrm{mm}^{3}\right)$. Após seis meses de acompanhamento, e descartadas causas secundárias para a citopenia, houve diminuição da contagem plaquetária $\left(40.000 / \mathrm{mm}^{3}\right)$ agora acompanhada de anemia (hemoglobina $=10,5 \mathrm{~g} / \mathrm{dl}$ ) normocítica, normocrômica, sem reticulocitose. No MO observou-se medula normocelular com diseritropoese discreta e redução do número de megacariócitos. Na BMO havia hiperplasia eritróide discreta. No cariótipo foi detectada deleção a do braço longo do cromossomo 11 [del(11q)]. Esta alteração cromossômica está associada ao uso de inibidores de topoisomerase, caracterizando assim o quadro de SMD-t.

Há muitas diferenças entre a SMD primária e secundária ou SMD-t. A secundária costuma acometer pacientes mais jovens em relação à primária (que é doença de indivíduo idoso), há maior taxa de transformação para leucemia aguda, as citopenias e a displasia de medula são mais severas, há menor celularidade da medula óssea com maior grau de fibrose e ocorre um maior número de alterações citogenéticas complexas ou duplas. Em geral, estes pacientes possuem prognóstico desfavorável em comparação com a SMD primária.

\section{Fisiopatologia e Citogenética}

Cerca de $80 \%$ dos pacientes com SMD-t apresentam anormalidades citogenéticas, tais como, -5 , del(5q), -7 , del(7q), del(13q), del(17p) e -18. Também apresentam maior incidência de mutações no gene P53 e aumento da instabilidade microssatélite, o que predispõe à instabilidade genética.

Os agentes quimioterápicos, como melphalan e ciclofosfamida, estão associados com o risco de SMD-t em um efeito dose dependente. Os agentes alquilantes podem causar deleções ou perda de todo o braço dos cromossomos 5 e 7.

Maior suscetibilidade a quebras na região do centrômero após a exposição a esses agentes alquilantes pode explicar as alterações genéticas observadas. Há duas vias bem descritas para a SMD-t após o uso desses agentes: a primeira está relacionada à del(7q) e -7, na qual ocorre mutação do gene RAS e hipermetilação da região promotora do P15 e a segunda envolve del(5q) e -5 em que pode aparecer mutação do gene P53.

\section{Neoplasias e Agentes Quimioterápicos}

Atualmente, o linfoma de Hodgkin é considerado uma das neoplasias hematológicas de maior índice de cura a depender do estágio inicial. Os protocolos mais utilizados para o tratamento costumam ser:ABVD, COPP/ABVD e BEACOPP. Josting e cols $^{4}$ avaliaram 5.411 casos tratados com estes esquemas quimioterápicos e detectaram uma incidência global de 0,08\% (46 pacientes) de SMD/LMA secundária ao tratamento. Dentre os casos relacionados aos esquemas ABVD, COPP/ABVD e BEACOPP, a incidência foi de 1, 30 e 11 casos respectivamente, sendo 10 casos de SMD e 32 casos de LMA.

Fenaux e cols ${ }^{1}$ avaliaram os fatores de risco para SMD-t e LMA secundária em mulheres tratadas para câncer de mama por um período de 16 anos, na França. Foram encontrados 44 casos de SMD-t e 138 LMAs secundárias. Estes casos foram estudados quanto ao regime quimioterápico utilizado e foi detectado um risco relativo de 14.1 para o uso de inibidores de topoisomerase, especialmente mitoxantrona, e de 2.9 para o uso de antracíclicos $(\mathrm{P}<0,05)$. Foi, portanto, demonstrado, pela primeira vez, que o risco do uso de inibidores de topoisomerase pode ser até maior que o de antracíclicos para o desenvolvimento de disfunção medular.

Atualmente, os protocolos para tratamento de câncer de mama têm utilizado epirrubicina (Epi) como droga adjuvante juntamente com maiores doses de ciclofosfamida (Ciclo). Praga e cols ${ }^{5}$ avaliaram 7.110 mulheres tratadas com Epi em combinação com Ciclo e detectaram incidência de SMD/LMA secundária de 0,55\%. Conforme aumentam-se as doses das duas substâncias, há aumento da incidência de SMD/LMA secundária com valores de $0,37 \%$, para doses de epi+ciclo $\leq 720 \mathrm{mg} / \mathrm{m}^{2}+<6.3 \mathrm{mg} / \mathrm{m}^{2}$, e de $4,97 \%$, para as doses superiores, demonstrando-se nítida correlação entre dose cumulativa e incidência de doença.

Knipp e cols ${ }^{2}$ avaliaram 2.603 pacientes alemães com SMD e encontraram $133(5,1 \%)$ casos relacionados à quimioterapia (QT) e/ou agentes imunossupressores e radioterapia (RT), sendo 61 homens e 72 mulheres com mediana de idade de 64 anos. Dentre as neoplasias primárias mais comuns foram encontrados: $16 \%$ mieloma múltiplo, $15 \%$ mama, $14 \%$ linfoma não-Hodgkin e 6\% linfoma de Hodgkin. Cinqüenta e três porcento dos pacientes receberam só QT, $20 \%$ receberam só RT e 27\% tratamento combinado, com período de latência de 70 meses, 126 meses e 51 meses, respectivamente, para o desenvolvimento da SMD-t, destacando-se a maior participação da QT como fator principal para o desenvolvimento da doença medular. A sobrevida média destes pacientes foi de nove 
meses enquanto a de SMD primária foi de 26 meses.

Recentemente, foram descritos casos de SMD secundária à azatioprina (Aza) e com interessante correlação com alterações citogenéticas, em especial envolvendo o cromossomo 7. Tais pacientes tinham mediana de idade de 47 anos, duração de tratamento de 83 meses (24-168m) e a quantidade de Aza acumulada de 253 g (27-765). Alterações citogenéticas envolvendo o cromossomo 7 estavam presentes em 9/12 pacientes avaliados. Knipp e cols ${ }^{3}$ sugeriram que o uso prolongado de Aza aumenta o risco de SMD secundária em torno de cem vezes em relação ao risco de SMD primária da população geral.

Os análogos de purina também foram associados ao desenvolvimento de SMD-t. Recentemente, um caso relacionado ao uso de cladribina para o tratamento de linfoma de baixo grau foi descrito em paciente de 68 anos de idade, com cariótipo normal.

Quimioterapia mieloablativa seguida de resgate com transplante autólogo de medula (TMO-A) é uma opção para o tratamento dos linfomas de alto e baixo grau. Tem sido o tratamento de escolha para os linfomas agressivos que apresentam recaída ou como terapia de consolidação para os linfomas indolentes. O problema desta alternativa terapêutica é o aparecimento de SMD/LMA secundária aos regimes de condicionamento. Estudos retrospectivos apresentam incidência variando desde 1\% de 12\% de SMD/LMA secundária após uma mediana de seguimento de seis anos. Essa diferença tão ampla pode ser explicada pelos diversos regimes utilizados, assim como pode estar relacionada aos esquemas de tratamento usados previamente pelos pacientes, posto que o efeito dos quimioterápicos está diretamente relacionado às doses empregadas.

Com o objetivo de avaliar de maneira mais precisa o impacto do TMO-A na incidência de SMD/LMA secundária, o grupo alemão para o estudo de linfoma de baixo grau fez uma análise randomizada para detectar tais efeitos. Quatrocentos e quarenta pacientes receberam 4-6 ciclos de esquema CHOP e depois foram randomizados para: braço A, mais dois ciclos de CHOP e manutenção com interferon ou braço B, mobilização com Dexa-BEAM seguida de irradiação corporal total e ciclofosfamida (60 mg/kg). Após mediana de seguimento de 45 meses, nenhum caso de SMD-t foi detectado no grupo A, mas foram observados cinco casos no braço B, perfazendo um risco estimado de 3,8\%, em cinco anos. Os cinco pacientes desenvolveram SMD após período de latência de 9, 20, 30, 36 e 51 meses. Dois pacientes (2/5) evoluíram para LMA após 40 e 44 meses de seguimento. Este foi o primeiro estudo randomizado a demonstrar um importante aumento de incidência de SMD secundária à QT em altas doses com resgate de células-tronco em pacientes com neoplasias hematológicas, chamando a atenção sobre o riscobenefício da realização de tais procedimentos em linfomas de baixo grau.

Dentre as opções terapêuticas para o tratamento das
SMD secundária à terapia, figuram o suporte transfusional e o uso dos agentes hipometilantes para os casos que se apresentam com alterações citogenéticas relacionadas ao cromossomo 7. O suporte transfusional ainda é uma terapêutica bastante utilizada pois prioriza a qualidade de vida destes pacientes, e a terapêutica hipometilante é capaz de postergar o tempo para transformação em leucemia aguda, porém a cura desta patologia de prognóstico extremamente desfavorável ainda parece distante da realidade atual.

\section{Abstract}

The leukaemogenic effect of chemotherapeutic agents after treatment for other malignancies have been well described. Myelodysplastic syndrome secondary to chemo- and radiotherapy (MDS-t) usually develops four to seven years after the initial exposure to chemotherapy frequently involving young patients, shows a high incidence of transformation to $A M L$, is associated with severe cytopenias, trilineage dysplasia, reduced marrow cellularity and fibrosis, and presents an incidence of chromosomal abnormalities of up to $80 \%$ of the cases. The most common abnormalities are related to chromosomes 5 and 7 . Alkylating agents have been considered the most common drugs associated with MDS-t. High dose chemotherapy used as part of the conditioning regimen prior to bone marrow transplantation as well as traditional regimens such as COPP/ABV and BEACOPP have also been associated with MDS-t. Recently, drugs such as azathioprine, rituximab and cladribine have been reported as causes too. Due to the increasing survival of patients suffering from other malignancies, MDS-t results as a mutagenic effect of these therapies and is related to poor prognosis. Rev. bras. hematol. hemoter. 2006;28(3):201-203.

Key words: Secondary myelodysplastic syndrome; therapy relatedMDS; chemotherapy-MDS; MDS-t.

\section{Referências Bibliográficas}

1. Fenaux P, Le Deley MC, Suzan F, Cutuli B, Delaloge S, Hill C. Risk factors for MDS and leukemia after breast cancer: results of a French multicenter case-control study. Leukemia Reserch 2005; 29(Sup 1).

2. Knipp S, Kundgen A, Hildebrandt B, Hass R, Gattermann N, Germing U. Characteristics of therapy-related MDS in 133 patients. Leukemia Reserch 2005;29 (Sup 1).

3. Knipp S, Hildebrandt B, Richter J, Hass R, Germing U, Gattermann N. Secondary MDS following treatment with azathioprine are associated with aberrations of chromosome 7. Haematologica 2005; 90(5):691-693.

4. Josting A, Wiedermann S, Franklin J, May M, Sieber M, Wolf J, et al. Secondary myeloid and myelodysplastic syndromes in patients treated for Hodgkin's disease: a report from the German Hodgkin's Lymphoma Study Group. J Clin Oncol 2003;21(18):3.440-6.

5. Praga C, Bergh J, Bliss J et al. Risk of acute myeloid leukemia and myelodysplastic syndrome in trials of adjuvant Epirrubicin for early breast cancer: correlation with doses of epirrubicin and cyclophosphamide. J Clinical Oncol 2005;18:4.179-91.

Avaliação: Um revisor externo e editorial board

Conflito de interesse: Não declarado

Recebido: 31/05/2006

Aceito após modificações: 11/09/2006 